conductivity, ${ }^{6}$ enhanced energy transfer, ${ }^{7}$ guest-tunable spin crossover, ${ }^{8}$ reversible ferromagnetic-antiferromagnetic behavior, ${ }^{9}$ resistance switching, ${ }^{10}$ and thermoelectric behavior. ${ }^{11}$

\section{Electronic conductivity}

As discussed elsewhere in this issue (see the Leong et al. article), MOFs with intrinsic electronic conductivity (i.e., in which charges move through the framework itself) are scarce. Strategies for synthesizing these build on the extensive knowledge base of conducting organic materials and nonporous coordination polymers. ${ }^{12,13}$ Valuable lessons also come from decades of research on electron-transfer processes in solution and in mixed-valence complexes, ${ }^{14-16}$ which show that electron transfer between metal centers in coordination complexes is governed by the extent of wave function mixing between the centers. The well-known Robin-Day classification of mixed-valence systems uses this concept to categorize the extent of electronic interaction between charge donor and acceptor sites, with Classes I, II, and III ranging, respectively, from isolated charges to fully delocalized charge. ${ }^{16}$ In most MOFs, there is little charge transfer between metal nodes in the structure (corresponding to Robin-Day Class I) because most are in redox-inactive states and are coordinated to ligands that are poor electron donors (e.g., carboxylates) and do not facilitate groundstate ligand-to-metal charge transfer. This is the case for HKUST-1 (from the Hong Kong University of Science and Technology), a MOF constructed of $\mathrm{Cu}$ (II) dimers bridged by four carboxylate groups. Electrical measurements show that the material is an insulator. ${ }^{17}$

One can envision that a properly chosen guest molecule could overcome this lack of communication by creating a bridge between metal centers, creating a donor-bridge-acceptor geometry. In mixed-valence complexes, Robin-Day Class II or borderline Class III materials often involve a bridging ligand. ${ }^{14}$ The charges are still localized, but can be induced to move if thermally activated. New bridge-to-metal charge-transfer optical transitions are also observed. We recently reported that infiltrating HKUST-1 with the electron-accepting molecule 7,7,8,8-tetracyanoquinodimethane (TCNQ) leads to an increase in electrical conductivity of more than seven orders of magnitude. ${ }^{6}$ A model for hopping transport enabled by superexchange mixing was proposed, in which TCNQ bridges copper dimers in the MOF (Figure 1a). ${ }^{6,11}$ This model is supported by experimental observations, including the temperature dependence of the conductivity, shifts in TCNQ vibrational bands, and new chargetransfer bands in the visible-near-infrared; the presence of new charge-transfer bands in particular is a known signature of ground-state mixing with excited states by superexchange mechanisms. ${ }^{14}$ Subsequent band-structure calculations predicted that empty TCNQ molecular orbitals are aligned such that the Fermi level moves close to the valence band of the MOF, creating a population of thermally accessible holes. This prediction is consistent with the measured Seebeck coefficient (relating the thermoelectric voltage induced by a temperature difference across a material), which identifies the carriers as holes. ${ }^{11}$

The dramatic change in electrical properties induced by TCNQ is perhaps the clearest example of how a guest molecule can create an emergent property in a MOF. It remains to be seen whether this specific Guest@MOF example can be implemented in other MOFs to create conducting materials. More generally, however, these results suggest a design concept - creating donor-bridge-acceptor units within a MOFwhich could be used by deliberately designing MOFs with binding sites that can accommodate bridging guest molecules. This is a difficult synthetic challenge, but one that could pay significant dividends if it can be achieved. However, a recent computational study provides guidance, showing that a number of copper paddlewheel MOFs have the right geometry for
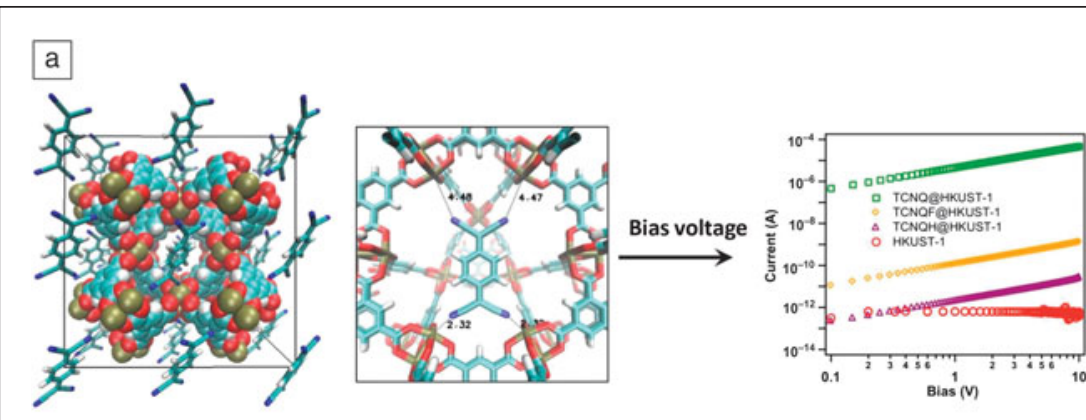

$\mathrm{b}$

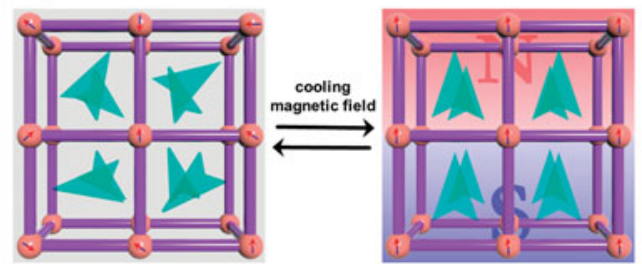

Figure 1. (a) Density functional theory-calculated (left) structure of $7,7,8,8$ tetracyanoquinodimethane (TCNQ) molecules bridging copper dimers in the pores of HKUST-1, forming a network of donor-bridge-acceptor complexes that facilitate charge transport (middle), with detailed lowest-energy structure; numbers are $\mathrm{Cu}-\mathrm{N}$ bond distances in Angstroms (element color code: carbon, turquoise; hydrogen, white; oxygen, red; nitrogen, blue; copper, bronze. (Right) Application of a bias voltage leads to current flow, the magnitude of which depends on the chemical nature of the guest molecule (TCNQ and derivatives in this case). Adapted with permission from Reference 5. (C) 2015 American Chemical Society. (b) Schematic view showing the simultaneous electric polarization and magnetization of perovskite structured Guest@MOFs materials through decreasing the temperature and applying a magnetic field. The cyan objects represent polar guest molecules. The parallel orientation of polar guest molecules is responsible for electrical ordering, and the alignment of magnetic orientations (red and blue arrows) is responsible for magnetic ordering in the material. Adapted with permission from Reference 6 (C) 2014 AAAS. Note: TCNQF, 2,3,5,6-tetrafluoro-7,7,8,8-tetracyanoquinodimethane; TCNQH, (cyclohexane-1,4-diylidene)dimalononitrile; HKUST, Hong Kong University of Science and Technology. 
TCNQ to bridge two copper dimers, as is thought to occur in HKUST-1. ${ }^{18}$

\section{Multiferroic behavior}

Multiferroics are materials that show the coexistence of at least two orders among magnetic, electric, and elastic properties. The materials that show both magnetic and electric ordering have a wide range of applications, especially in spintronics, data storage, and sensors, due to the possibility of four-state memory. ${ }^{19}$ Coupling between magnetic and electric ordering could allow control over the magnetization through the application of an electric field and vice versa (magnetoelectric [ME] effect)..$^{20}$ These applications have been widely explored in inorganic perovskites $\left(\mathrm{ABX}_{3}\right.$ where $\mathrm{A}$ is an $n$-valent and $\mathrm{B}$ is an $(n+2)$-valent cation, and $\mathrm{X}$ is an anionic atom) such as $\mathrm{BiFeO}_{3}$, where the $d$-electrons of $\mathrm{Fe}(\mathrm{III})$ are the origin of magnetic behavior and the coupling between the $2 p$ orbital of an oxygen atom with empty bismuth $6 p$ orbitals caused ferroelectric behavior and exhibited antiferromagnetic performance at room temperature. ${ }^{21}$ However, the parameter space for tailoring such inorganic oxide materials is limited due to constricted atomic variations that are possible from the periodic table.

In recent years, coordination polymers/ MOFs have been investigated as promising alternatives with organic-inorganic duality of the perovskite $\mathrm{ABX}_{3}$ structure (Figure 1b). Judicious choice of subunits from variable guest molecules (A), metal nodes (B), and linkers $(\mathrm{X})$ provides new opportunities to bring appropriate physical and chemical properties in the same material. The best-known multiferroic MOFs are (DMA) $\left[\mathrm{M}(\mathrm{HCOO})_{3}\right]$ (DMA $=$ dimethylammonium, $\mathrm{Me}_{2} \mathrm{NH}_{2}{ }^{+}$; $\mathrm{M}=\mathrm{Fe}, \mathrm{Mn}, \mathrm{Co}, \mathrm{Ni}$, and $\mathrm{Me}=$ methyl group), which were recently reported. ${ }^{22}$ The orientation of the cationic DMA guest molecules hosted in the pores of the anionic MOF is the main reason for electric ordering in these materials, where the dynamically disordered dipolar moments of the guest molecules at room temperature become cooperatively ordered at lower temperatures and assist in the generation of electric polarization in the material. The unpaired electrons of transition-metal atoms are the origin of magnetic behavior through unpaired $d$-electrons (Figure 1b). Although the mechanisms of these magnetic and electric orderings are considered to be independent, recent studies show significant cross-coupling (ME effect). ${ }^{23}$ This could be explained by the electric-field manipulation of the orientation of H-bonding between the cationic guest molecules with the framework. ${ }^{24-27}$ However, due to the low transition temperatures for electrical ordering $(160-185 \mathrm{~K})$ and magnetic behavior $(8-36 \mathrm{~K}),{ }^{21}$ the ME coupling effects are limited to low transition temperatures (up to $19 \mathrm{~K}$ ). ${ }^{25-27}$ This low transition temperature could be due to the weak magnetic interaction between the metal ions, so there is a great need for research focused on the transition temperature of the materials and use of elements with a higher magnetic moment, such as lanthanides. Recent developments in organic multiferroic materials, ${ }^{28,29}$ which could act as the guest molecules, would also boost the field.

\section{Exciton harvesting}

In organic materials, excitons can be formed by light absorption, charge injection, or ionization (Figure 2), enabling their use as active components of photovoltaic cells, in light-emitting diodes (LEDs), and for radiation detection. These applications are well established, with large classes of both molecular and

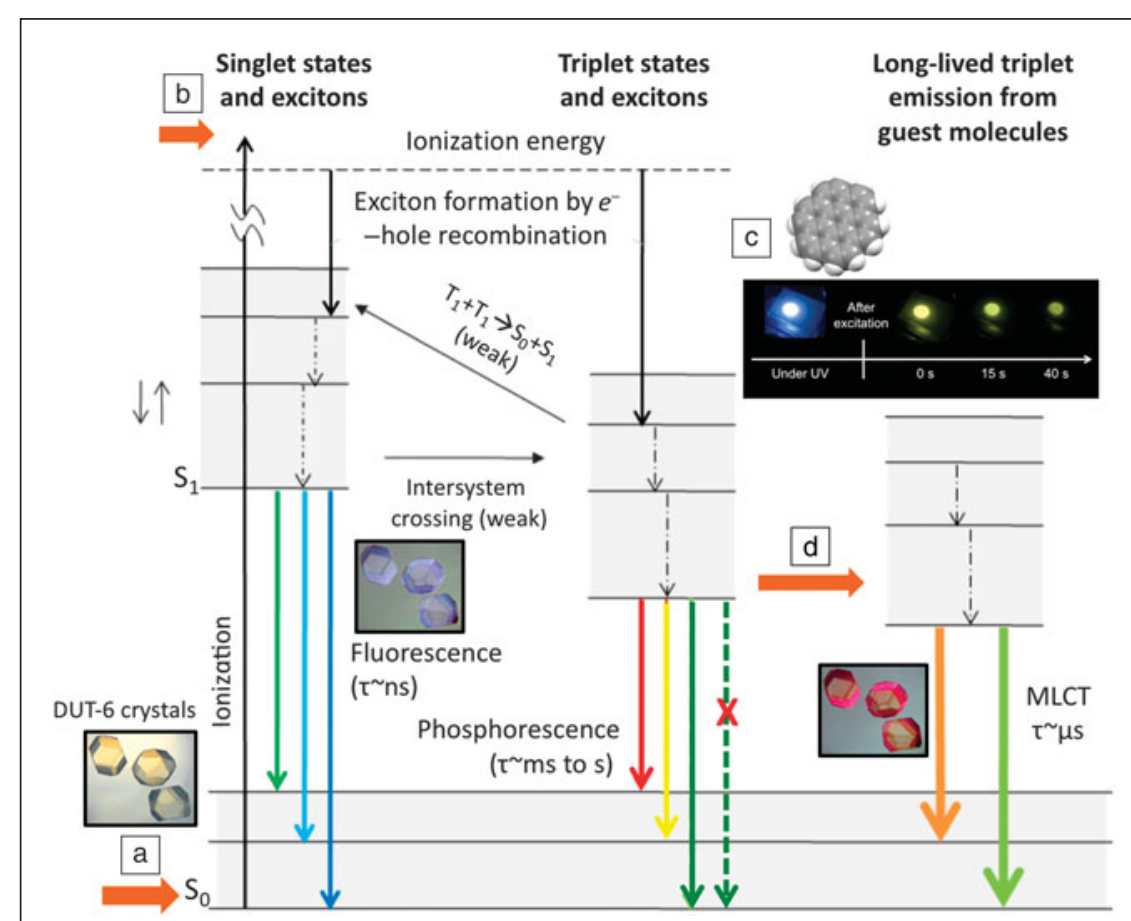

Figure 2. Energy-level diagram illustrating the ways in which metal-organic frameworks (MOFs) can be used for exciton capture and conversion. Colors of the arrows are a qualitative indication of the wavelength of the emitted light. (a) Typical MOFs, such as crystals of DUT- 6 are transparent, but emit blue to near-ultraviolet (UV) fluorescence from their lowest singlet-excited state $S_{1}$ (violet crystals). These frameworks can also be used as hosts for fused aromatic compounds and other emitters that, (b) when excited by charge injection, form both singlet and triplet excitons. Triplet excitons emit long-lived phosphorescence in the visible and are useful for light-emitting diode applications. (c) The quantum efficiency and emission lifetime of such emitters are increased by immobilizing the emitter in the MOF pores, which reduces the rate of nonradiative decay pathways (dashed green arrow), as seen here for coronene. Note: gray spheres, carbon; white spheres, hydrogen. (Inset) Photos: blue singlet emission and yellow phosphorescence as a function of time. (d) Alternatively, ionization of the MOF (black arrow at left) also leads to singlet and triplet excitons; the latter can be efficiently utilized for radiation detection by incorporating organometallic triplet-harvesting complexes in MOF pores. Pink crystals: (triplet harvester)@DUT-6. Note: DUT, Dresden University of Technology; MLCT, metalligand charge transfer; $S_{0}$, ground electronic state; $S_{1}$, first singlet-excited state; $T_{1}$, lowest triplet-excited state; $\tau$, emission lifetime. 
polymeric materials designed for these purposes. However, in most cases, layers of these materials are disordered when incorporated into the device, leading to problems such as aggregation, fast luminescence quenching, short triplet-state lifetimes, and device instability. ${ }^{30,31}$ The Guest@MOF concept offers several potential advantages as a novel class of materials for addressing these problems.

\section{MOFs as hosts for LED emitter molecules}

Molecules with long-lived phosphorescence (emission from triplet-excited states) are desirable for LEDs. However, the phosphorescence can be partially or completely quenched by nonradiative pathways. For example, coronene emits blue fluorescence in solution at room temperature, but emits only yellow phosphorescence at low temperatures, where vibrational motion is minimized..$^{32}$ Dissolving the molecule in an organic polymer enables phosphorescence to be observed at room temperature. However, emitter loading is limited by aggregation, and polymers of interest for this purpose, such as poly(methyl methacrylate) (PMMA), are heat and air sensitive. In contrast, it was recently demonstrated that room-temperature phosphorescence can be observed by encapsulating coronene in the zeolitic imidazolate framework (ZIF) MOF known as ZIF-8 (coronene@ZIF-8, Figure 2c). This MOF also allows much higher loadings without aggregation and enables phosphorescence at temperatures as high as $460 \mathrm{~K}$. Remarkably, coronene@ZIF-8 is also stable in air for periods of at least one month, even though its pore openings are large enough to emit oxygen molecules. ${ }^{32}$

\section{Triplet harvesting for LEDs}

The Guest@MOF concept can be extended by encapsulating light-emitting molecules within the pores of one of the many luminescent MOFs. ${ }^{33,34}$ The luminescence of the resulting composite can be tuned by varying the identity and concentration of the guest as well as the linker, allowing either white light or tunable color to be produced. ${ }^{35,36}$ Increases in both quantum yield and temperature stability have been observed as well. The process is illustrated in Figure $2 \mathrm{~b}$ and $\mathrm{d}$. Charge injection into luminescent linker molecules creates singlet and triplet excitons. Singlet excitons with lifetimes on the order of 1-10 ns emit light in the blue or near UV. To achieve overall higher quantum efficiency and enable the color of the emitted light to be tuned, organometallic complexes of heavy elements such as $\mathrm{Ru}, \mathrm{Os}$, and Ir are infiltrated into the MOF pores (Figure 2d). These molecules scavenge the linker-based triplet excitons via the heavy-atom effect, leading to visible emission from the guest on time scales of $\mathrm{ms}$ to $\mathrm{s} .{ }^{37}$ For many of the same reasons discussed in the previous section, MOFs represent an attractive host of triplet-harvesting molecules, providing a thermally stable matrix that inhibits aggregation.

\section{Radiation detection}

We discovered several years ago that luminescent MOFs can also be radioluminescent; that is, interaction with ionizing radiation (particles with energies $>10 \mathrm{eV}$ ) leads to fast pulses of light known as scintillation. ${ }^{38,39}$ This light is emitted from the decay of singlet excitons formed when ions recombine with electrons and holes. Typically, fast light emission is desirable for radiation-detection applications to avoid interference with particle detection. However, as only $25 \%$ of the excitons are singlets, $75 \%$ of the available excitation is lost (analogous behavior is found in LEDs). A logical solution to this problem is to infiltrate the MOF pores with triplet-harvesting molecules, analogous to LEDs. However, we also found that infiltrating a radioluminescent MOF, such as IRMOF-8 (isoreticular MOF), with small amounts of a triplet-harvesting complex creates emission of both fast light pulses from the decay of singlet excitons, produced by the MOF linker, and slower ones produced by the decay of triplet excitons via the guest molecule. The intensity ratio of the emission at these two different wavelengths depends on the identity of the particle. Light particles, such as electrons, yield a higher ratio of singlet-to-triplet light, whereas heavier particles such as protons more effectively quench the singlet emission. This phenomenon represents a new radiation-detection mechanism that has advantages of simplicity and speed over conventional particle discrimination mechanisms that rely on analysis of the time signatures of the emitted radioluminescence. In addition to MOFs, ${ }^{5}$ this approach can also be implemented in polymers. $^{40}$

\section{Conclusions, challenges, and new directions}

The Guest@MOF concept provides unique opportunities to achieve properties that are uncharacteristic of either the MOF or the guest, and which encompass applications including energy harvesting, sensing, microelectronics, and light emission. However, much remains to be understood for this concept to be realized in practical devices. Immediate challenges that we foresee are the extent to which guest molecules can be confined within MOF pores without loss during subsequent processing steps. The role of other "bystander" guests, such as solvent molecules, is not understood, although we have evidence that these can affect the conductivity of TCNQ@HKUST-1.5

Additionally, the range of guest-framework interaction energies is considerably wider than that typically seen in the storage of light gases by MOFs, in which weak dispersion forces dominate. As a result, conventional force-field approaches to modeling Guest@MOF materials are unlikely to be useful. This presents a considerable challenge for theorists, as accurate electronic structure modeling of the large unit cells in MOFs (HKUST-1 has $>600$ atoms in its unit cell, for example) is computationally costly. Synthesis can be a challenge as well; for example, designing or modifying a MOF family to allow for efficient synergy with an interesting class of guests is often not straightforward. Nevertheless, it is clear that Guest@MOF opens new avenues to MOF design and extension to unexpected applications, presenting many opportunities to design functional materials based on this remarkable class of materials. 


\section{Acknowledgments}

R.M. would like to thank the Alexander von Humboldt Foundation for a postdoctoral fellowship. M.A. acknowledges the Sandia National Laboratory Directed Research and Development Program. Sandia National Laboratories is a multiprogram laboratory managed and operated by Sandia Corporation, a wholly owned subsidiary of Lockheed Martin Corporation, for the US Department of Energy's National Nuclear Security Administration under Contract DE-AC04-94AL85000.

\section{References}

1. M.P. Suh, H.J. Park, T.K. Prasad, D.-W. Lim, Chem. Rev. 112, 782 (2012).

2. J.R. Li, R.J. Kuppler, H.C. Zhou, Chem. Soc. Rev. 38, 1477 (2009).

3. L.E. Kreno, K. Leong, O.K. Farha, M. Allendorf, R.P. Van Duyne, J.T. Hupp, Chem. Rev. 112, 1105 (2012).

4. J.W. Liu, L.F. Chen, H. Cui, J.Y. Zhang, L. Zhang, C.Y. Su, Chem. Soc. Rev. 43, 6011 (2014).

5. M.D. Allendorf, M.E. Foster, F. Leonard, V. Stavila, P.L. Feng, F.P. Doty, K. Leong, E.Y. Ma, S.R. Johnston, A.A. Talin, J. Phys. Chem. Lett. 6, 1182 (2015). 6. A.A. Talin, A. Centrone, A.C. Ford, M.E. Foster, V. Stavila, P. Haney, R.A. Kinney, V. Szalai, F. El Gabaly, H.P. Yoon, F. Leonard, M.D. Allendorf, Science 343, 66 (2014).

7. K. Leong, M.E. Foster, B.M. Wong, E.D. Spoerke, D.V. Gough, J.C. Deaton, M.D. Allendorf, J. Mater. Chem. A 2, 3389 (2014).

8. J.J.M. Amoore, S.M. Neville, B. Moubaraki, S.S. Iremonger, K.S. Murray, J.F. Letard, C.J. Kepert, Chem. Eur. J. 16, 1973 (2010).

9. M. Kurmoo, H. Kumagai, K.W. Chapman, C.J. Kepert, Chem. Commun. 24, 3012 (2005). doi: 10.1039/B500614G.

10. L. Pan, G. Liu, H. Li, S. Meng, L. Han, J. Shang, B. Chen, A.E. Platero-Prats, W. Lu, X.D. Zou, R.W. Li, J. Am. Chem. Soc. 136, 17477 (2014).

11. K.J. Erickson, F. Léonard, V. Stavila, M.E. Foster, C.D. Spataru, R.E. Jones, B.M. Foley, P.E. Hopkins, M.D. Allendorf, A.A. Talin, Adv. Mater. 27, 3453 (2015).

12. D.M. D'Alessandro, J.R.R. Kanga, J.S. Caddy, Aust. J. Chem. 64, 718 (2011).

13. L. Sun, M.G. Campbell, M. Dincă, Angew. Chem. Int. Ed. 55, 3566 (2016).

14. B.S. Brunschwig, C. Creutz, N. Sutin, Chem. Soc. Rev. 31, 17 (2002).

15. R.A. Marcus, N. Sutin, Biochim. Biophys. Acta 811, 265 (1985).

16. M.B. Robin, P. Day, "Mixed Valence Chemistry-A Survey and Classification," in Advances in Inorganic Chemistry and Radiochemistry, H.J. Emeléus, A.G. Sharpe, Eds. (Academic, New York, 1967), p. 248.

17. S.S.-Y. Chui, S.M.-F. Lo, J.P.H. Charmant, A.G. Orpen, I.D. Williams, Science 283, 1148 (1999).

18. X.W. Nie, A. Kulkarni, D.S. Sholl, J. Phys. Chem. Lett. 6, 1586 (2015).

19. M. Gajek, M. Bibes, S. Fusil, K. Bouzehouane, J. Fontcuberta, A. Barthelemy, A. Fert, Nat. Mater. 6, 296 (2007).

20. S.-W. Cheong, M. Mostovoy, Nat. Mater. 6, 13 (2007).

21. G. Rogez, N. Viart, M. Drillon, Angew. Chem. Int. Ed. 49, 1921 (2010).

22. P. Jain, V. Ramachandran, R.J. Clark, H.D. Zhou, B.H. Toby, N.S. Dalal, H.W. Kroto, A.K. Cheetham, J. Am. Chem. Soc. 131, 13625 (2009).

23. A. Stroppa, P. Jain, P. Barone, M. Marsman, J.M. Perez-Mato, A.K. Cheetham, H.W. Kroto, S. Picozzi, Angew. Chem. Int. Ed. 50, 5847 (2011).

24. R.I. Thomson, P. Jain, A.K. Cheetham, M.A. Carpenter, Phys. Rev. B Condens. Matter 86, 214304 (2012).

25. Y. Tian, J. Cong, S. Shen, Y. Chai, L. Yan, S. Wang, Y. Sun, Phys. Status Solidi Rapid Res. Lett. 8, 91 (2014).

26. Y. Tian, S. Shen, J. Cong, L. Yan, S. Wang, Y. Sun, J. Am. Chem. Soc. 138 , 782 (2016).

27. Y. Tian, A. Stroppa, Y. Chai, L. Yan, S. Wang, P. Barone, S. Picozzi, Y. Sun, Sci. Rep. 4, 6062 (2014).

28. W. Qin, B. Xu, S. Ren, Nanoscale 7, 9122 (2015).

29. S. Horiuchi, Y. Tokunaga, G. Giovannetti, S. Picozzi, H. Itoh, R. Shimano, R. Kumai, Y. Tokura, Nature 463, 789 (2010).

30. A. Hagfeldt, G. Boschloo, L.C. Sun, L. Kloo, H. Pettersson, Chem. Rev. 110, 6595 (2010).

31. Q. Wang, D. Ma, Chem. Soc. Rev. 39, 2387 (2010).

32. H. Mieno, R. Kabe, N. Notsuka, M.D. Allendorf, C. Adachi, Adv. Opt. Mater. 4, 1015 (2016), http://www.dx.doi.org/10.1002/adom.201600103.
33. M.D. Allendorf, C.A. Bauer, R.K. Bhakta, R.J.T. Houk, Chem. Soc. Rev. 38, 1330 (2009).

34. Y.J. Cui, Y.F. Yue, G.D. Qian, B.L. Chen, Chem. Rev. 112, 1126 (2012).

35. C.-Y. Sun, X.-L. Wang, X. Zhang, C. Qin, P. Li, Z.-M. Su, D.-X. Zhu, G.-G. Shan, K.-Z. Shao, H. Wu, J. Li, Nat. Commun. 4 (2013).

36. W. Xie, W.W. He, D.Y. Du, S.L. Li, J.S. Qin, Z.M. Su, C.Y. Sun, Y.Q. Lan, Chem. Commun. 52, 3288 (2016).

37. M. Thompson, MRS Bull. 32, 694 (2007).

38. F.P. Doty, C.A. Bauer, A.J. Skulan, P.G. Grant, M.D. Allendorf, Adv. Mater. 21, 95 (2009)

39. J.J. Perry, P.L. Feng, S.T. Meek, K. Leong, F.P. Doty, M.D. Allendorf, J. Mater. Chem. 22, 10235 (2012).

40. P.L. Feng, J. Villone, K. Hattar, S. Mrowka, B.M. Wong, M.D. Allendorf, F.P. Doty, IEEE Trans. Nucl. Sci. 59, 3312 (2012).

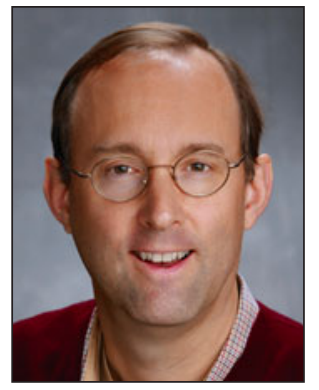

Mark D. Allendorf is Director of the Hydrogen Advanced Materials Research Consortium and a senior scientist at Sandia National Laboratories. He received an $A B$ degree in chemistry from Washington University in St. Louis and a PhD degree in chemistry from Stanford University. His research focuses on the fundamental science and applications of metal-organic frameworks and related materials. He has been published in more than 160 publications, including more than 120 journal articles. He is President Emeritus and Fellow of The Electrochemical Society and his awards include a 2014 R\&D 100 Award for a novel approach to radiation detection. Allendorf can be reached by phone at 925-294-2895 or by email at mdallen@ sandia.gov.

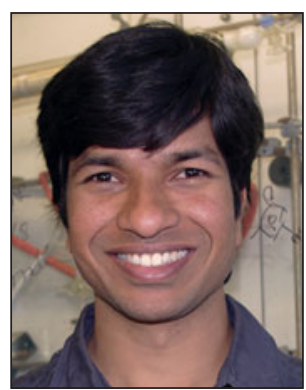

Raghavender Medishetty is an Alexander von Humboldt Fellow at Technische Universität München, where he currently studies nonlinear optical properties of metal-organic frameworks. He received his master's degree from Banaras Hindu University, India. He later moved to the National University of Singapore (NUS) as a Presidential Graduate Fellow and studied solidstate photochemistry in metal complexes and coordination polymers. He received the Outstanding Researcher Award during his graduate studies from the Department of Chemistry at NUS. Medishetty can be reached by phone at +49 (0) $1521-073-1732$ or by email at raghavender.medishetty@tum.de.

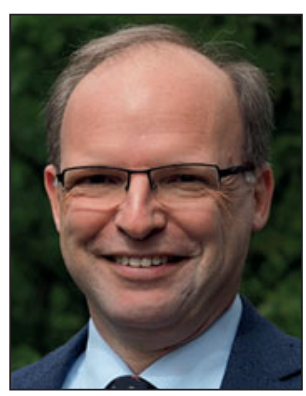

Roland A. Fischer holds the Chair of Inorganic and Metal-Organic Chemistry at Technische Universität München (TUM). He was a full professor of inorganic chemistry at RuhrUniversität Bochum from 1998 to 2015, and associate professor at Heidelberg University from 1996 to 1997. He obtained Habilitation (1995) and Dr. rer. nat. (1989) degrees from TUM, where he also studied chemistry (1981-1987). His research interests include organometallic clusters and nanoparticles for catalysis, precursor chemistry for chemical vapor deposition, and the supramolecular chemistry and property tailoring of metal-organic frameworks. Fischer can be reached by phone at +49 (0) 89-289 13081 or by email at roland.fischer@ ch.tum.de. 